\title{
SCATTERING FROM RANDOM LINEAR ARRAYS WITH CLOSEST APPROACH*
}

\author{
$\mathrm{BY}$ \\ Z. A. MELZAK \\ The University of British Columbia, Vancouver, Canada
}

1. Probability distributions for the echo returned from a random linear scattering array are of some importance in several different situations, especially in connection with the use of radar and sonar. In the simplest case $n$ identical isotropic point scatterers are placed at random on the interval $[0, L]$, the source of radiation is at the point $(-s, 0)$ which is sufficiently far from the origin so that the ratio $\left(s+x_{1}\right) /\left(s+x_{2}\right)$ is sufficiently close to 1 for any two points $x_{1}$ and $x_{2}$ on $[0, L]$, and the length $L$ is an integral multiple of the wave-length $\lambda$. The problem of finding the probability density for the components of the scattered signal reduces then to finding the joint probability density $W_{n}(X, Y)$ for the components of the vector

$$
\phi=\left(\sum_{i=1}^{n} \cos \left(2 \pi x_{i} / \lambda\right), \sum_{j=1}^{n} \sin \left(2 \pi x_{j} / \lambda\right)\right),
$$

where each variable $x_{i}$ is taken from the uniform rectangular probability distribution: $\operatorname{Pr}\left(x_{i} \leq x\right)=x / L, 0 \leq x \leq L$. The probability density for the amplitude of the scattered signal is obtained in terms of the probability density $W_{n}(R)$ for the quantity $R=$ $\left(X^{2}+Y^{2}\right)^{1 / 2}$. It follows that aside from some normalization factor the problem is formally equivalent to the classical isotropic plane random walk.

If the scatterers are spheres of radius $r$, it may still be convenient to regard them as points, but with the additional restriction that no two points are closer than $2 r$. This assumption also fits the case where each scatterer has a radius of repulsion, within which no other scatterer may enter. We shall consider here the corresponding scattering problem: to find the probabilities $W_{n}(X, Y)$ and $W_{n}(R)$ under the restriction that the $n$ point scatterers on $[0, L]$ are not allowed to be closer to each other than $a, 0 \leq a \leq$ $L /(n-1)$, and are otherwise at random. Our approach will be mainly geometrical, and we shall begin by finding the sample space of the configurations.

2. We start with a well known problem in elementary geometrical probability: on the segment $[0, L] n$ points are taken at random, given a number $a, 0 \leq a \leq L /(n-1)$, what is the probability $P(n, a, L)$ that no two points are closer than $a$ ? If $n=2$, then the sample space of pairs of points $\left(x_{1}, x_{2}\right), 0 \leq x_{1}, x_{2} \leq L$, is the square of side $L$; let $D$ be its diagonal through the origin, and draw the two lines parallel to $D$ at the distance $2^{-1 / 2} a$ from it. The hexagonal subset of the square, contained between these two lines, is then the sample space of the forbidden configurations $\left(x_{1}, x_{2}\right)$ with $\left|x_{1}-x_{2}\right| \leq a$; the remainder of the square consists of two triangles which can be moved together to form a square of side length $L-a$. Hence, by the randomness assumption, $P(2, a, L)=(L-a)^{2} / L^{2}=(1-a / L)^{2}$.

The general case can be handled in the same way. In the $n$-dimensional Euclidean space $E^{n}$ we consider a Cartesian coordinate system with the $n$ axes $X_{1}, \cdots, X_{n}$. Let $H$ be the hypercube

$$
H=\left\{\left(x_{1}, \ldots, x_{n}\right): 0 \leq x_{i} \leq L, i=1, \ldots, n\right\} ;
$$

${ }^{*}$ Received July 5, 1961; revised manuscript received November 3, 1961. 
$H$ is the sample space of all $n$-tuples of points on the segment $[0, L]$. Let $I_{i}$ be the interval $[0, L]$ on the $X_{i}$-axis, and in the two-dimensional square face $S_{i j}=I_{i} \times I_{j}$ let $D_{i j}$ be the diagonal through the origin. Let $B_{i j}$ be the hexagonal subset of $S_{i j}$, consisting of all points no further from $D_{i j}$ than $2^{-1 / 2} a$. Let $F_{i j}$ be the Cartesian product of $B_{i i}$ and all $I_{k}, k \neq i, j$. That is,

$$
F_{i j}=\left\{\left(x_{1}, \cdots, x_{n}\right):\left(x_{i}, x_{i}\right) \epsilon B_{i j}, 0 \leq x_{k} \leq L, k \neq i, j\right\} .
$$

Then $F_{i j}$ is the sample space of those $n$-tuples $\left(x_{1}, \cdots, x_{n}\right)$ for which $\left|x_{i}-x_{i}\right| \leq a$. Hence the sample space of the allowed configurations, that is, of all $n$-tuples $\left(x_{1}, \cdots, x_{n}\right)$ such that $\left|x_{i}-x_{i}\right| \geq a$ for all $i$ and $j, i \neq j$, is the set

$$
H-\bigcup_{1 \leq i<i \leq n} F_{i i} \text {. }
$$

Therefore by the randomness assumption

$$
P(n, a, L)=\operatorname{vol}\left(H-\bigcup_{1 \leq i<j \leq n} F_{i i}\right) / \operatorname{vol} H .
$$

When the $\left(\begin{array}{c}n \\ 2\end{array}\right)$ sets $F_{i j}$ are removed from $H$, the remainder $H-\cup_{1 \leq i<i \leq n} F_{i j}$ consists of $n$ ! congruent simplexes. These can be assembled by a sequence of translations so as to form an $n$-dimensional hypercube $H^{\prime}$ of edge-length $L-(n-1) a$. The required translations are as follows: for $i=1, \cdots, n$ let $\xi_{i}$ denote the vector in $E^{n}$ whose $i$-th coordinate is $-a$ and all others are 0 , now translate by $\xi_{i}$ (by $\xi_{i}$ ) every one of the $n ! / 2$ simplexes, which lies on the same side of $F_{i j}$ as the positive half of the $X_{i}$-axis (the $X_{i}$-axis), and carry out this operation for all $i$ and $j, 1 \leq i<j \leq n$. We have now by (1) $P(n, a, L)=\operatorname{vol} H^{\prime} / \operatorname{vol} H$, and therefore

$$
P(n, a, L)=[1-(n-1) a / L]^{n},
$$

which is the well known solution of the problem, [1], [2]. An equivalent way of expressing it is the following: let $n$ points $x_{1}, \cdots, x_{n}$ be placed at random on the interval $[0, L]$, let $u=\min _{i<i}\left|x_{i}-x_{j}\right|$ be the nearest approach of any two of the $n$ points, and let $P_{n}(u, L)$ be the probability density for $u$; then

$$
P(n, a, L)=\int_{a}^{L /(n-1)} P_{n}(u, L) d u,
$$

and so by (2) we obtain

$$
\begin{aligned}
P_{n}(u, L) & =[n(n-1) / L][1-(n-1) u / L]^{n-1}, & & 0 \leq u \leq L /(n-1), \\
& =0 & & L /(n-1)<u .
\end{aligned}
$$

The $k$-th moment of $u$ is

$$
\mu_{n, k}=\int_{0}^{L /(n-1)} u^{k} P_{n}(u, L) d u=\left[\left(\begin{array}{c}
n+k \\
k
\end{array}\right)\right]^{-1}[L /(n-1)]^{k},
$$

the mean is $L /\left(n^{2}-1\right)$ and the standard deviation is $\left[L /\left(n^{2}-1\right)\right][n /(n+2)]^{1 / 2}$. It may be observed that if $n \rightarrow \infty$ and $a \rightarrow 0$ so that $n^{2} a \rightarrow \alpha, 0<\alpha<\infty$, then

$$
\lim P(n, a, L)=P(\alpha, L)=\exp (-\alpha / L) ;
$$


and if $n \rightarrow \infty$ and $L \rightarrow \infty$ so that $L / n^{2} \rightarrow \beta, 0<\beta<\infty$, then

$$
\lim P(n, a, L)=P(\beta, a)=\exp (-a / \beta), \lim \mu_{n, k}=k ! \beta^{k} .
$$

We observe further that if $a \ll L$ then (2) may be written as

$$
P(n, a, L)=1-2\left(\begin{array}{l}
n \\
2
\end{array}\right) a / L+O\left(a^{2} / L^{\prime}\right) .
$$

We consider next the analogous problem for $k$-tuple clumping: $n$ points are taken at random on the interval $[0, L]$, an integer $k$ and a number $a$ are given, where $2 \leq k \leq n$ and $0<a<L$, what is the probability $P(k, n, a, L)$ that no $k$ points lie on an interval of length $a$ ? Equivalently, if a segment of length $a$ is dragged along $[0, L], P(k, n, a, L)$ is the probability that this segment never covers more than $k-1$ points at a time Again, if $n$ events are to occur at random times during the time interval $\left[\begin{array}{ll}0, & T\end{array}\right]$ $P(k, n, \tau, T)$ is the probability that no $k$ events occur within a time interval of length $\tau$. This problem is more difficult than the previous one, but by a similar geometrical reasoning it can be shown that if $a \ll L$ then

$$
P(k, n, a, L)=1-k\left(\begin{array}{l}
n \\
k
\end{array}\right)(a / L)^{k-1}+O\left(a^{k} / L^{k}\right) .
$$

An outline of the proof follows. Let $H$ be the same hypercube as before, and let the sets $I_{1}, \cdots, I_{n}$ be as previously defined. Let $i_{1}, i_{2}, \cdots, i_{k}$ be $k$ integers, such that $1 \leq i_{1}<i_{2}<\cdots<i_{k}$, then $S_{i_{1} i_{2} \ldots i_{k}}=I_{i_{1}} \times I_{i_{2}} \times \cdots \times I_{i_{k}}$ is one of the $\left(\begin{array}{l}n \\ k\end{array}\right) k$ dimensional faces of $H$, which contain the origin. Let $D_{i_{1} i_{2} \ldots i_{k}}$ be the longest diagonal of the $k$-dimensional hypercube $S_{i_{1} i_{2} \ldots i_{k}}$, passing through the origin. Let $K(p)$ denote the $k$-dimensional hypercube of edge-length $a$, whose center is the point $p$, and whose edges are parallel to the coordinate axes $X_{i_{1}}, X_{i_{2}}, \cdots, X_{i_{k}}$. In analogy to the previously considered sets $B_{i j}$, define

$$
B_{i_{1} i_{2} \cdots i_{k}}=\left(S_{i_{1} i_{2} \cdots i_{k}}\right) \cap\left(\bigcup_{p \ell D i_{1} i_{2} \cdots i_{k}} K(p)\right),
$$

and let $F_{i_{1} i_{2} \ldots i_{k}}$ be the Cartesian product of $B_{i_{1} i_{2} \ldots i_{k}}$ with all the $I_{j}$ 's, $j \neq i_{1}, i_{2}, \cdots, i_{k}$. That is,

$$
\begin{aligned}
F_{i_{1} i_{2} \cdots i_{k}}=\left\{\left(x_{1}, x_{2}, \cdots, x_{n}\right):\left(x_{i_{2}}, x_{i_{2}}, \cdots, x_{i_{k}}\right) \epsilon B_{i_{1} i_{2} \cdots i_{k}},\right. \\
\left.0 \leq x_{i} \leq L, j \neq i_{1}, i_{2}, \cdots, i_{k}\right\} .
\end{aligned}
$$

Then $F_{i_{1} i_{2} \ldots i_{k}}$ is the sample space of all $n$-tuples $\left(x_{i}, \cdots, x_{n}\right)$, for which the points corresponding to $x_{i_{1}}, x_{i_{2}}, \cdots, x_{i_{k}}$ are in the forbidden configuration, that is, are covered by a segment of length $\leq a$. Hence by the randomness assumption

$$
P(k, n, a, L)=\operatorname{vol}\left(H-\cup F_{i_{1} i_{2} \ldots i_{k}}\right) / \operatorname{vol} H,
$$

where the union is taken over all the $\left(\begin{array}{l}n \\ k\end{array}\right)$ selections of the integers $i_{1}, i_{2}, \cdots, i_{k}$. By using the inclusion-exclusion principle, [3], and some elementary volume estimates, it may be shown that

$$
\operatorname{vol}\left(H-\cup F_{i_{1} i_{2} \cdots i_{k}}\right)=\operatorname{vol} H-\sum \operatorname{vol} F_{i_{i_{2}} \cdots i_{k}}+O\left(a^{k} / L^{k}\right) .
$$


Since there are $\left(\begin{array}{l}n \\ k\end{array}\right)$ sets $F_{i_{1} i_{2} \ldots i_{k}}$, and since the volume of each one is $L^{n-k}\left[k L a^{k-1}+O\left(a^{k}\right)\right]$, (5) follows at once.

A different generalization of the first problem is the following: let $A=\left(a_{i j}\right)$ be an $n \times n$ real matrix with $a_{i e}=a_{j i}>0$ for $1 \leq i<j \leq n$, and $a_{i i}=0, i=1, \cdots, n, n$ labelled points $x_{1}, \cdots, x_{n}$ are placed at random on the segment $[0, L]$; what is the probability $P(n, A, L)$ that $\left|x_{i}-x_{i}\right| \geq a_{i i}$ for all $i$ and $j$ ? Here it may be shown, [4], that if the matrix $A$ satisfies the triangular condition

$$
a_{i j}+a_{i k} \geq a_{i k}, \quad 1 \leq i, j, k \leq n,
$$

then

$$
P(n, A, L)=1 / n ! \sum_{\sigma \varepsilon G}\left[\max \left(0,1-1 / L \sum_{k=1}^{n-1} a_{\sigma(k) \sigma(k+1)}\right)\right]^{n},
$$

where $G$ is the symmetric group on $n$ elements, whose members are

$$
\sigma=\left(\begin{array}{cccc}
1 & 2 & \cdots & n \\
\sigma(1) \sigma(2) & \cdots & \sigma(n)
\end{array}\right)
$$

If $a_{i j} \ll L$ for all $i$ and $j$, then in analogy to (4) and (5) we have

$$
P(n, A, L)=1-2 / L \sum_{i<i} a_{i i}+O\left[\max a_{i i} / L\right]^{2} .
$$

3. We take up now the scattering problem mentioned at the end of section 1. For simplicity it will be assumed throughout that $\lambda=2 \pi$. If the assumption concerning the closest approach of two scatterers were absent, then by the standard methods, for instance, by that in [5], we could have shown that

$$
W_{n}(X, Y)=(2 \pi)^{-2} \int_{-\infty}^{\infty} \int_{-\infty}^{\infty} \exp \left[-i\left(\rho_{1} X+\rho_{2} Y\right)\right] A_{n}\left(\rho_{1}, \rho_{2}\right) d \rho_{i} d \rho_{2},
$$

where

$$
A_{n}\left(\rho_{1}, \rho_{2}\right)=1 / L^{n} \int_{0}^{L} \cdots \int_{0}^{L} \exp i\left(\rho_{1} \sum_{1}^{n} \cos x_{k}+\rho_{2} \sum_{1}^{n} \sin x_{k}\right) d x_{1} \cdots d x_{n} .
$$

We notice that the region of integration in (7) is the hypercube $H$ of section 2. Recalling that the sample space of the forbidden configurations $\left(x_{1}, \cdots, x_{n}\right)$, with $\left|x_{i}-x_{i}\right| \leq a$ for some $i$ and $j$, is the set $F=\bigcup_{i<i} F_{i j}$, and the sample space of the allowed configurations is therefore the residual set $R=H-F$ of the hypercube $H$, we have the following: under the assumption that no two $x$ 's are closer than $a$, and otherwise the $x$ 's are at random, the probability $W_{n}(X, Y)$ is still given by (6) but in the integral for $A_{n}\left(\rho_{1}, \rho_{2}\right)$ the region of integration is $R$, not $H$. That is,

$$
W_{n}(X, Y)=(2 \pi)^{-2} \int_{-\infty}^{\infty} \int_{-\infty}^{\infty} \exp \left[-i\left(\rho_{1} X+\rho_{2} Y\right)\right] B_{n}\left(\rho_{1}, \rho_{2}\right) d \rho_{1} d \rho_{2},
$$

where

$$
B_{n}\left(\rho_{1}, \rho_{2}\right)=(\operatorname{vol} R)^{-1} \int \underset{R}{\ldots} \int \exp i\left(\rho_{1} \sum_{1}^{n} \cos x_{k}+\rho_{2} \sum_{1}^{n} \sin x_{k}\right) d x_{1} \cdots d x_{n} .
$$


Most of our work from now on will be concerned with estimating $B_{n}\left(\rho_{1}, \rho_{2}\right)$. We shall assume that $a$ is small, and we shall derive a formula of the type

$$
B_{n}\left(\rho_{1}, \rho_{2}\right)=B_{0}\left(\rho_{1}, \rho_{2}\right)+a B_{1}\left(\rho_{1}, \rho_{2}\right)+O\left(a^{2}\right) ;
$$

$B_{0}$ and $B_{1}$ will be found, and since the final aim is finding the Fourier transform (8) of $B_{n}$, it will be necessary to examine the dependence of the $O\left(a^{2}\right)$ term in (10) on $\rho_{1}$ and $\rho_{2}$. This will complicate somewhat the derivation of the succeeding estimates.

4. Let $S_{1}, \cdots, S_{N}$ be a finite number of sets in the Euclidean space $E^{n}$, let $S$ be their union, and let $f$ be an integrable function defined on $S$. Then

$$
\int_{S} f d V=\sum_{i=1}^{N} \int_{S_{i}} f d V-\sum_{1 \leq i<i \leq N} \int_{S_{i} \cap S_{j}} f g_{i j} d V,
$$

where $g_{i j}$ is a function defined on $S_{i} \cap S_{i}$ and $0 \leq g_{i i} \leq 1$, for each $i$ and $j$. To prove (11), we consider the set of $2^{N}$ atoms, corresponding to the sets $S_{i}$. These atoms are defined as follows: let $\bar{S}_{i}=S-S_{i}$, and let $K\left(S_{i}\right)$ stand for either one of the sets $S_{i}$ and $\bar{S}_{i} ;$ then the $2^{N}$ atoms are all the $2^{N}$ sets of the form

$$
K\left(S_{1}\right) \cap K\left(S_{2}\right) \cap \cdots \cap K\left(S_{N}\right) .
$$

An atom which is not the empty set, is said to be of order $k$ if it lies in exactly $k$ different sets $S_{i}$. Define now $g_{i i}$ as follows: $g_{i j}(x)=2 / k$ if $x \varepsilon A$ and $A$ is an atom of order $k$. Since such an atom lies in exactly $\left(\begin{array}{l}k \\ 2\end{array}\right)$ sets $S_{i} \cap S_{i}$ the corresponding contribution in (11) from the first sum is $k \int_{A} f d V$, and the contribution from the second sum is $(k-1) \int \mathrm{A} f d V$; since $S$ is the union of all the $2^{N}$ atoms, the equation (11) balances.

It may be mentioned that a formula more general than (11) is

$$
\begin{array}{r}
\int_{S} f d V=\sum_{i=1}^{N} \int_{S_{i}} f d V-\sum_{1 \leq i_{1}<i_{2} \leq N} \int_{S_{i_{1}} \cap S_{i_{2}}} f d V+\sum_{1 \leq i_{1}<i_{2}<i_{3} \leq N} \int_{S_{i_{2}} \cap S_{i_{2}} \cap S_{i_{3}}} f d V-\cdots \\
+(-1)^{r+1} \sum_{1 \leq i_{1}<i_{2}<\cdots<i_{r} \leq N} \int_{S_{i_{1}} \cap \cdots \cap S_{i_{2}}} f g_{i_{i_{2}} \cdots_{i_{r}}} d V,
\end{array}
$$

where $g=g_{i_{1} i_{2} \ldots i_{r}}$ is defined on the set $S_{i_{1}} \cap S_{i_{2}} \cap \cdots \cap S_{i_{r}}$, and $0 \leq g \leq 1$. This formula can be proved in the same way as (11); it would be the proper starting point if, instead of deriving (10), we were interested in deriving the superior approximation

$$
B_{n}\left(\rho_{1}, \rho_{2}\right)=B_{0}\left(\rho_{1}, \rho_{2}\right)+a B_{1}\left(\rho_{1}, \rho_{2}\right)+\cdots+a^{r} B_{r}\left(\rho_{1}, \rho_{2}\right)+O\left(a^{r+1}\right) .
$$

We proceed to apply (11) in order to derive (10). Recall that

$$
R=H-F=H-\bigcup_{1 \leq i<i \leq n} F_{i i},
$$

and let $T$ stand for the integrand in (9). Then

$$
\int_{R} T d V=\int_{H} T d V-\int_{F} T d V
$$

we now apply (11) with $N=\left(\begin{array}{l}n \\ 2\end{array}\right)$ and with the sets $F_{i j}$ as the $S_{i}$ 's. We obtain then

$$
\int_{F} T d V=\sum_{1 \leq i<i \leq n} \int_{F_{i j}} T d V-\sum \int_{F_{i j} \cap F_{r s}} T g_{i j r s} d V,
$$


where the second summation is over all the distinct

$$
\left(\begin{array}{c}
n \\
2
\end{array}\right)=\left(n^{4}-2 n^{3}-n^{2}+2 n\right) / 8
$$

pairs $(i, j),(r, s)$ with $1 \leq i<j \leq n$ and $1 \leq r<s \leq n$.

5. We shall derive in this section an estimate of the first sum in (13). By the definition of $F_{i j}$ as a product set, and by the multiplicative property of the integrand $T$, we have

$$
\begin{aligned}
\int_{F_{i j}} T d V=\left[\int_{0}^{L}\right. & \left.\cdots \int_{0}^{L} \exp i\left(\rho_{1} \sum_{1}^{n-2} \cos x_{k}+\rho_{2} \sum_{1}^{n-2} \sin x_{k}\right) d x_{1} \cdots d x_{n-2}\right] \\
& \cdot\left[\int_{B_{j i}} \exp i\left[\rho_{1}(\cos x+\cos y)+\rho_{2}(\sin x+\sin y)\right] d x d y\right],
\end{aligned}
$$

where $B_{i j}$ is the hexagon in the $x y$-plane, with the vertices $(0,0),(0, a),(a, 0),(L, L)$, $(L-a, L),(L, L-a)$. By adding onto $B_{i j}$ the two triangles with the vertices $(0,0)$ $(a, 0),(0,-a)$ and $(L, L),(L-a, L),(L, L+a)$ we complete the hexagon to the parallelogram given by $x-a \leq y \leq x+a, 0 \leq x \leq L$. Therefore, denoting by $J$ the second integral on the right hand side in (15), we have

$$
J=\int_{0}^{L} \int_{x-a}^{x+a} \exp i\left[\rho_{1}(\cos x+\cos y)+\rho_{2}(\sin x+\sin y)\right] d y d x+c_{1} a^{2}
$$

where $\left|c_{1}\right| \leq 1$. We observe that

$$
\int_{x-a}^{x+a} f(y) d y=2 a f(x)+c_{2} M a^{2}
$$

where $\left|c_{2}\right| \leq 1$ and $M=\max \left|f^{\prime}(y)\right|$. Applying this to (16), with

$$
f(y)=\exp i\left(\rho_{1} \cos y+\rho_{2} \sin y\right),
$$

we have $\left|f^{\prime}(y)\right| \leq \rho$, where

and therefore

$$
\rho=\left(\rho_{1}^{2}+\rho_{2}^{2}\right)^{1 / 2}
$$

$$
J=\int_{0}^{L}\left[2 a \exp 2 i\left(\rho_{1} \cos x+\rho_{2} \sin x\right)+c_{2} \rho a^{2} \exp i\left(\rho_{1} \cos x+\rho_{2} \sin x\right)\right] d x+c_{1} a^{2} .
$$

It was assumed that $L$ is an integral multiple of the wave-length $\lambda=2 \pi$, therefore

$$
J=2 a L J_{0}(2 \rho)+c_{2} \rho a^{2} L J_{0}(\rho)+c_{1} a^{2},
$$

where $J_{0}(x)$ is the Bessel function of the first kind and order 0 , and $\rho$ is given by (17). The $(n-2)$-fold integral in (15) is likewise expressed in terms of the Bessel functions:

$$
\int_{0}^{L} \cdots \int_{0}^{L} \exp i\left(\rho_{1} \sum_{1}^{n-2} \cos x_{k}+\rho_{2} \sum_{1}^{n-2} \sin x_{k}\right) d x_{1} \cdots d x_{n-2}=\left[L J_{0}(\rho)\right]^{n-2} .
$$

This, together with (18), gives an estimate on the first sum in (13):

$$
\begin{aligned}
\sum_{1 \leq i<j \leq n} \int_{F_{i j}} T d V=\left(\begin{array}{l}
n \\
2
\end{array}\right) & \\
& \cdot \int_{F_{i j}} T d V=\left(\begin{array}{l}
n \\
2
\end{array}\right) a L^{n-2}\left[J_{0}(\rho)\right]^{n-2}\left[2 L J_{0}(2 \rho)+c_{2} a \rho L J_{0}(\rho)+c_{1} a\right] .
\end{aligned}
$$


6. We consider next the terms of the second sum in (13). These are of two kinds: (a) $N_{1}$ terms coming from the pairs $F_{i i}, F_{r s}$ with all four indices distinct, and (b) $N_{2}$ terms coming from the pairs $F_{i j}, F_{r s}$ where two indices coincide, that is, $i=r$ or $j=s$ or $j=r$.

For a term $K$ of the first kind we have, by the mean value theorem for integrals,

$$
K=\int_{F_{i} \cap F_{r \cdot}} T g_{i j r s} d V=c_{3} \int_{F_{i j} \cap F_{r}} T d V
$$

where $\left|c_{3}\right| \leq 1$, since $0 \leq g_{i i r s} \leq 1$. Now, by the definition of the sets $F_{i i}$ and by the multiplicative property of the integrand $T$, we have

$$
K=c_{3}\left[L J_{0}(\rho)\right]^{n-4} J^{2},
$$

with $J$ given by (18). Therefore

$$
K=c_{3} a^{2}\left[L J_{0}(\rho)\right]^{n-4}\left[2 L J_{0}(2 \rho)+c_{2} \rho a L J_{0}(\rho)+c_{1} a\right]^{2} .
$$

A typical term of the second kind is

$$
\int_{F_{i j} \cap F_{i},} T g_{i j i s} d V
$$

here by a method similar to that one used in section 5 , we derive the estimate

$$
\int_{F_{i,} \cap F_{i}} T g_{i i i s} d V=c_{4}\left[L J_{0}(\rho)\right]^{n-3}[a(\rho+a)]^{2},
$$

where $c_{4}$ is a constant.

Putting together the results of this section and the previous one, in particular, by (19), (20) and (21), we get from (12)

$$
\begin{aligned}
\int_{R} T d V & =\int_{H} T d V-\int_{F} T d V=\left[L J_{0}(\rho)\right]^{n}-\left(\begin{array}{c}
n \\
2
\end{array}\right) a\left[L J_{0}(\rho)\right]^{n-2}\left[2 L J_{0}(2 \rho)\right. \\
& \left.+c_{2} a \rho L J_{0}(\rho)+c_{1} a\right]+N_{1} c_{3} a^{2}\left[L J_{0}(\rho)\right]^{n-4}\left[2 L J_{0}(2 \rho)+c_{2} a \rho L J_{0}(\rho)+c_{1} a\right]^{2}
\end{aligned}
$$

Actually, we do not need every term explicitly, and we write (22) as

$$
\int_{R} T d V=\left[L J_{0}(\rho)\right]^{n}-n(n-1) a L^{n-1} J_{0}^{n-2}(\rho) J_{0}(2 \rho)+c a^{2} E,
$$

where $c$ is a constant, and the error term $E$ is a finite sum of expressions like

$$
\rho^{i} J_{0}^{n-k}(\rho) J_{0}^{p}(2 \rho), \quad 0 \leq j \leq 2, \quad 0 \leq k \leq 4, \quad 0 \leq p \leq 2 .
$$

Hence (9) becomes

$$
B_{n}\left(\rho_{1}, \rho_{2}\right)=[L-(n-1) a / L]^{-n}\left[L^{n} J_{0}^{n}(\rho)-n(n-1) a L^{n-1} J_{0}^{n-2}(\rho) J_{0}(2 \rho)+c a^{2} E\right],
$$

and expanding the negative power, we get

$$
B_{n}\left(\rho_{1}, \rho_{2}\right)=J_{0}^{n}(\rho)+n(n-1)(a / L) J_{0}^{n-2}(\rho)\left[J_{0}^{2}(\rho)-J_{0}(2 \rho)\right]+c^{\prime}(a / L)^{2} E^{\prime},
$$

where $c^{\prime}$ is a constant and $E^{\prime}$ is of the same type as $E$. This is the expression of the type (10) that we have been looking for.

7. In taking the inverse Fourier transform (8) of $B_{n}\left(\rho_{1}, \rho_{2}\right)$ we observe that the contribution from the term $c^{\prime}(a / L)^{2} E^{\prime}$ is $0\left(a^{2} / L^{2}\right)$, since for large $\rho$ we have $J_{0}^{m}(\rho)=$ 
$O\left(\rho^{-m / 2}\right)$, and since each term in $E^{\prime}$ involves a sufficiently high power of $J_{0}(\rho)$ to compensate for the presence of the powers of $\rho$ (a tacit assumption is made here that $n$ is sufficiently large). Hence by (8)

$$
\begin{gathered}
W_{n}(X, Y)=(2 \pi)^{-2} \int_{-\infty}^{\infty} \int_{-\infty}^{\infty} \exp \left[-i\left(\rho_{1} X+\rho_{2} Y\right)\right] J_{0}^{n}(\rho) d \rho_{1} d \rho_{2}+(2 \pi)^{-2}(a / L) n(n-1) \\
\cdot \int_{-\infty}^{\infty} \int_{-\infty}^{\infty} \exp \left[-i\left(\rho_{1} X+\rho_{2} Y\right)\right] J_{0}^{n-2}(\rho)\left[J_{0}^{2}(\rho)-J_{0}(2 \rho)\right] d \rho_{1} d \rho_{2}+O\left(a^{2} / L^{2}\right) .
\end{gathered}
$$

We introduce the polar coordinates:.

$$
X=R \cos \varphi, \quad Y=R \sin \varphi, \quad \rho_{1}=\rho \cos \alpha, \quad \rho_{2}=\rho \sin \alpha,
$$

and (25) becomes

$$
\begin{aligned}
& W_{n}(R)=W_{n}(R \cos \varphi, R \sin \varphi)=(2 \pi)^{-2} \\
& \cdot \int_{0}^{\infty} \int_{0}^{2 \pi} \exp [-i \rho R \cos (\varphi-\alpha)] J_{0}^{n}(\rho) \rho d \alpha d \rho+(2 \pi)^{-2}(a / L) n(n-1) \\
& \cdot \int_{0}^{\infty} \int_{0}^{2 \pi} \exp [-i \rho R \cos (\varphi-\alpha)] J_{0}^{n-2}(\rho)\left[J_{0}^{2}(\rho)-J_{0}(2 \rho)\right] \rho d \alpha d \rho+O\left(a^{2} / L^{2}\right)
\end{aligned}
$$

the integration over $\alpha$ can be carried out, and we get finally

$$
\begin{aligned}
W_{n}(R)=(2 \pi)^{-1} \int_{0}^{\infty} J_{0}(\rho R) J_{0}^{n}(\rho) \rho d \rho+n(n-1)(a / 2 \pi L) \\
\cdot \int_{0}^{\infty} J_{0}(\rho R) J_{0}^{n-2}(\rho)\left[J_{0}^{2}(\rho)-J_{0}(2 \rho)\right] \rho d \rho+O\left(a^{2} / L^{2}\right) .
\end{aligned}
$$

For $a=0$ this reduces, as it should, to the classical solution of the plane isotropic random walk, obtained by Kluyver, [6].

8. In conclusion, we shall formulate a general problem, which includes as special cases all those considered here and many more besides, for instance, the case of the so called hard-sphere gas model in the statistical mechanics. Let $n$ and $N$ be two positive integers and let $B$ and $P$ be two sets in the Euclidean space $E^{n} . P$ is assumed to have a center of symmetry $x$, and it may be thought of as being much smaller than $B . N$ points $x_{1}, \cdots, x_{N}$ are taken at random in $B$, and $P\left(x_{i}\right)$ is the set obtained by translating $P$ so that the center of symmetry is at $x_{i}$. The sample space of the points $x_{1}, \cdots, x_{N}$, or of the $N$-tuple $\left(x_{1}, \cdots, x_{N}\right)$, is the $N$-fold Cartesian product $\left\{\left(x_{1}, \cdots, x_{N}\right): x_{i} \varepsilon B\right.$, $i=1, \cdots, N\}$. We call an $N$-tuple a forbidden configuration if $x_{i} \varepsilon P\left(x_{j}\right)$ for some two members $x_{i}, x_{i}$ of the $N$-tuple, otherwise it is called an allowed configuration. The sample space $A$ of all allowed configurations is then

$$
A=\left\{\left(x_{1}, \cdots, x_{N}\right): x_{i} \notin P\left(x_{j}\right), 1 \leq i<j \leq N\right\} .
$$

Let $f(x)$ be an integrable function defined over $B$; our problem is that of evaluating the integral

$$
I=\int \underset{\boldsymbol{A}}{\ldots} \int f\left(x_{1}\right) \cdots f\left(x_{N}\right) d x_{1} \cdots d x_{N},
$$

where $d x_{i}$ is the volume element in $E^{n}$. The problem may be further extended by taking 
integrands of the type $F\left(x_{1}, \cdots, x_{N}\right)$, and also by letting the points $x_{1}, \cdots, x_{N}$ be distributed in $B$ according to some probability law. In the latter case the integral $I$ depends on that law, and we may ask, for instance, for its expected value.

While a general solution of this problem does not appear feasible, a possible approach might be to introduce a 'basic ratio', such as, for instance, the ratio of the diameters, widths or volumes, of the sets $P$ and $B$, and to find the coefficients in the expansion of $I$ in the powers of that ratio.

9. Drs. E. N. Gilbert and S. P. Lloyd of the Bell Telephone Laboratories, Murray Hill, N. J., have offered criticism and valuable suggestions. The work reported upon was started at the Signal Analysis Group, Massachusetts Institute of Technology, it was continued at the Bell Telephone Laboratories, and it was completed at the 1961 Summer Research Institute of the Canadian Mathematical Congress. The author wishes to express his thanks to these individuals and institutions.

\section{REFERENCES}

1. E. N. Gilbert and H. O. Pollak, Coincidences in Poisson patterns, The Bell Syst. Tech. J., 39, 1005-1034 (1957)

2. E. C. Molina, The theory of probability and some applications to engineering problems, Trans. A. I. E. E., 44, 294-299 (1925)

3. J. Riordan, An introduction to combinatorial analysis, Wiley, 1958

4. Z. A. Melzak, A problem in geometrical probability, Canadian Math. Bull., 4, 185-186 (1961)

5. S. Chandrasekhar, Stochastic processes in physics and astronomy, Rev. Mod. Phys., 15, 1-91 (1943)

6. G. N. Watson, Theory of Bessel functions, Cambridge, 1921. 